J Am Geriatr Soc. 2016 April ; 64(4): 739-751. doi:10.1111/jgs.14036.

\title{
Association Between Oral Health and Cognitive Status: A Systematic Review
}

\author{
Bei Wu, PhD ${ }^{a}$, Gerda G. Fillenbaum, PhD $^{b}$, Brenda L. Plassman, $\mathrm{PhD}^{\mathrm{b}, \mathrm{c}}$, and Liang Guo, \\ BS $^{a}$ \\ aSchool of Nursing, Duke University, Durham, NC \\ ${ }^{b}$ Center for the Study of Aging and Human Development, Duke University Medical Center, \\ Durham, NC \\ 'Department of Psychiatry and Behavioral Sciences, Duke University Medical Center, Durham, \\ NC
}

\section{Abstract}

OBJECTIVES-We have carried out a systematic review of longitudinal studies examining the association between oral health and cognitive decline.

DESIGN—Studies, published 01/1993-03/2013, were identified by search of English language publications in PubMed/Medline using relevant MeSH terms and title/abstract keywords, and from CINAHL using relevant subject headings. After applying eligibility criteria, and adding four studies identified from article references, 56 of the 1412 articles identified remained: 40 were cross-sectional, and 16 longitudinal; 11 of the latter examined the impact of oral health on change in cognitive health or dementia incidence, five examined the reverse.

SETTING-Sources of information included administrative data, subject evaluations in parent studies, medical and dental records, self-reports, and in-person evaluations.

MEASUREMENTS-Most studies used subjects whose oral or cognitive status was known, adding the missing piece using standard measures. The oral health information most frequently studied included number of teeth, periodontal and caries problems, and denture use. Cognition was most frequently evaluated using the MMSE, or by determination of dementia.

RESULTS-Some studies found that oral health measures such as number of teeth and periodontal disease were associated with increased risk of cognitive decline or incident dementia, while others did not find the association. Similarly, cognitive decline was not consistently associated with greater loss of teeth or number of caries. Methodological limitations likely play a major role in explaining the inconsistent findings.

CONCLUSION-It is unclear how or whether oral health and cognitive status are related. Additional research is needed in which there is greater agreement on how oral health and cognitive states are assessed, in order to better examine the linkages between these two health outcomes.

Corresponding author: Bei Wu, PhD, School of Nursing and Global Health Institute, Duke University, 307 Trent Drive, DUMC 3322, Durham, NC 27710, bei.wu@ duke.edu. Alternate corresponding author: Gerda G. Fillenbaum, PhD, 


\section{Keywords}

Oral health; dementia; cognitive decline

\section{INTRODUCTION}

In the past two decades, an increasing number of studies has examined the relationship between cognitive impairment and oral health in older adults, since question has arisen as to whether, in the elderly, there is an association between oral health status and cognitive status, possibly through a common inflammatory pathway. ${ }^{1,2}$ This issue is of considerable relevance given the rapid increase in the proportion and number of older persons in the population, an increasing number of whom are retaining their natural teeth. ${ }^{3}$ Further, approximately $36 \%$ of those over age 70 are cognitively impaired, ${ }^{4}$ and the prevalence of cognitive impairment and dementia increases with age. ${ }^{5} \mathrm{Up}$ to 5.1 million Americans currently have a dementing disorder, ${ }^{6}$ with an expected doubling by $2050 .{ }^{7}$

Concomitantly, evidence from clinical samples suggests that the elderly have an increased incidence of oral disease, the frequency of oral health problems increasing significantly in cognitively impaired elderly, particularly those with dementia. Any intervention that might help delay the onset or progression of dementia, including improvement of oral hygiene and dental services, could have a significant impact on personal and family well-being, and health care costs.

In order to examine the association between oral health and cognition in the elderly, and provide direction for future studies, we conducted a systematic review of the existing literature. Our focus is on longitudinal studies, since they may be informative with respect to causation.

\section{METHODS}

\section{Search Strategy}

The time frame considered was from the first relevant publication found in $1993,{ }^{8}$ to 03/01/2013. We searched for human study publications in English in the PubMed/Medline database, using the MeSH terms (Memory Disorders or Cognition or Cognition Disorders or Dementia) AND (Oral Health or Mouth Diseases or Tooth Diseases), which identified 515 articles; a title/abstract keyword search using (mouth or tooth or oral AND cogni* or Alzheimer or dement*), which identified 790 articles; and the CINAHL database using the subject headings (dementia+ or Alzheimer's diseases or cognition+ or cognition disorders+ or memory disorders+) AND (mouth diseases or tooth loss or dental caries), identifying 107 articles. After removing 41 duplicates from the 1412 articles identified, 1371 unique articles remained (Figure 1).

We applied two exclusionary criteria to identify non-qualifying articles. The first criterion excluded 1,273 articles that, by title/abstract, did not examine the association between cognition and oral health diseases and conditions in adults; this left 98 articles. The second criterion excluded articles that, based on review of the abstract by two readers, had 
inadequate information on oral health or cognitive status. Any disagreement was resolved by a third reviewer. In consequence, an additional 46 articles were excluded and 52 retained. To these were added four articles culled from article reference lists, resulting in a total of 56 articles, based on 55 studies. Of these, 40 articles represented cross-sectional studies, and 16 represented longitudinal studies.

\section{Data Extraction}

Two reviewers independently extracted information from each longitudinal article on: first author and date of publication, study name, location; study date(s), sample demographics; cognitive and oral health measures; covariates; and key findings. Disagreements on data extraction were resolved by consensus with the assistance of a third person.

\section{RESULTS}

The characteristics of the 16 longitudinal studies are summarized in Table 1. The first section summarizes the 11 studies of oral health predicting cognitive decline, the second section summarizes the five studies of cognitive status/dementia predicting oral health.

Several developed countries are represented: U.S. $(n=8)$, Japan $(n=3)$, Australia $(n=1)$, France $(\mathrm{n}=1)$, Denmark $(\mathrm{n}=1)$, Sweden $(\mathrm{n}=1)$, and a European consortium $(\mathrm{n}=1)$. Two studies used the same sample, but different outcomes and statistical analyses. ${ }^{9,10}$ All but four studies ${ }^{9-12}$ were conducted among community residents, but some community-based studies may have included nursing home residents. Sample sizes ranged from small $(\mathrm{N}=42)^{13}$ to large ( $\mathrm{N}=11,140$, from 215 centers in 20 European countries). ${ }^{14}$

Subject selection and source of data reflected several creative, overlapping approaches. These included use solely of administrative data, ${ }^{15}$ use of data already gathered in longitudinal studies (often designed for other purposes), ${ }^{9,10,16-20}$ selecting subjects for whom cognitive status or oral health status had been established and adding an oral or cognitive evaluation, ${ }^{8,11,13,21,22}$ and linking subject data to administrative records. ${ }^{23}$

Study design varied: intervention studies of good oral care; ${ }^{12,24}$ samples matched on demographics, by randomization, $, 12,13,21$ or through use of propensity scores; ${ }^{24}$ and natural history studies. $^{9-11,14-17,19,20,22,23}$

\section{Measures of Oral Health Status (information is summarized in on-line Table S1.)}

Of the 16 studies, nine used oral health evaluation, and seven used dental records. In studies gathering oral health data, examiners were trained, calibrated if more than one participated, and reliability determined for assessing a variety of oral health measures. The amount of oral health information gathered in person or from records, and used in analysis, varied from extensive and highly detailed, particularly in studies of oral health predicting cognitive impairment (number of decayed, missing, filled teeth; plaque; periodontal disease), ${ }^{8,13,20,22}$ to minimal (self-reported approximate number of teeth currently or when younger). ${ }^{14,15,19,23}$ Subject-provided information included pain or discomfort, ${ }^{17}$ bleeding gums, ${ }^{14}$ and denture use. ${ }^{11,19,23}$ Information from dental records was used in three studies, ${ }^{9,10,24}$ two of which relied on up to 40 years of records. ${ }^{9}, 10,25$ 
Tooth loss was the most common measure of oral health, recorded in 14 of the 16 studies, with a significant relationship to cognition reported in $11 .^{9-11,13,15-17,19,20,22,23}$ When used in analysis, the number of teeth present was typically categorized, with categorization varying from study to study, and sometimes linked to denture use. $9,11,15,17$

Eleven studies used clinical evaluation to assess oral health status. ${ }^{8-13,16,17,20,21,24}$ In some studies, standard oral health indices were used: Debris Index for plaque score, ${ }^{12}$ Community Periodontal Index, ${ }^{17}$ Gingival Index, ${ }^{20}$ Plaque Index, ${ }^{20}$ Katz criteria for roots, and adjusted caries increment, ${ }^{21}$ and National Institute for Dental Research (NIDR) criteria. $^{8,13,21,22}$

In examining periodontal disease, attention was paid to ${ }^{1)}$ extent of gingival bleeding on probing (ratio of examined sites), ${ }^{2)}$ proportion of examined sites with loss of attachment $>3$ $\mathrm{mm}$, and 3) mean pocket depth. Periodontal diseases were assessed in five studies, four on the impact of oral health on cognitive impairment (three studies used clinical assessment, ${ }^{16,17,20}$ one used self-report ${ }^{14}$ ) and in one study of the effect of cognitive impairment on oral health. ${ }^{13}$ In addition, two studies assessed periodontal damage based on decades of dental records. ${ }^{9,10}$

\section{Measures of Cognitive Assessment (information is summarized in on-line Table S2.)}

Assessment of the presence of dementia included self-reported diagnosis, use of standard clinical diagnostic criteria, neuropathological diagnostic criteria, and information from medical, dental, and administrative records. Cognitive status was based on a variety of neuropsychology measures, ranging from use of a substantial battery to reliance on a single brief measure.

The standard diagnostic criteria for dementia included DSM-III-R, DSM-IV, ICD-9CM-R, ICD-10, reflecting the date of evaluation. ${ }^{13-15,17,22}$ At times these criteria were supplemented by NINCDS-ADRDA (Alzheimer's disease [AD]), ${ }^{13,15,17}$ NINDS-AIREN (vascular dementia), ${ }^{15}$ Hachinski scale (vascular dementia), ${ }^{17}$ Lund \& Manchester (frontal lobe dementia), ${ }^{15}$ Geriatric Mental State examination, ${ }^{17}$ and the Global Deterioration Scale, ${ }^{26}$ which assesses both presence and severity of dementia. ${ }^{21,22}$ Neuropathological criteria ${ }^{27}$ were also used, but available for only one dataset. ${ }^{9}$

Non-AD dementias ${ }^{15,17,22}$ were rarely analyzed separately because of limited numbers. The validity of administrative records, which were frequently used, was evaluated for only one study. ${ }^{15}$ Both cognitive batteries and individual measures were used to assess cognitive change and ascertain the presence of dementia. The most popular single measure was the

Conflict of Interest: The editor in chief has reviewed the conflict of interest checklist provided by the authors and has determined that the authors have no financial or any other kind of personal conflicts with this paper.

Author contributions:

Substantial contributions to conception/design, acquisition of data, analysis and interpretation of data: Bei Wu, Gerda G. Fillenbaum, Brenda L. Plassman, Liang Guo

2. Drafting the article or revising it critically for important intellectual content: Bei Wu, Gerda G. Fillenbaum, Brenda L. Plassman, Liang Guo

3. 
Mini-Mental State Exam (MMSE), ${ }^{28}$ used in 10 studies - in eight as a single measure, four of which examined the effect of oral condition on cognitive state, ${ }^{12,14,16,19}$ and four the reverse, ${ }^{8,13,21,22}$ and in two studies as part of a larger neuropsychology battery. ${ }^{9}, 17$ All neuropsychology measures had established validity and reliability. Criteria used to assess cognitive change included self-perceived deterioration, change in score on neuropsychology measures, a specific decline (which differed across studies), crossing a cut-point (with cutpoints differing across studies), or reaching an administrative criterion indicating need for assistance.

\section{Covariates Used in Analysis (summarized in online Table S3.)}

The covariates used varied widely across studies. They included demographic characteristics (sex, race, education, income, -- none uniformly reported); and specific health conditions and associated risk factors or the number of health conditions present (12 studies), and the Charlson Index. ${ }^{31}$ Also considered were biomarker and genetic information (interleukin (IL)-6, C-reactive protein, APOE $\varepsilon 4,1^{\text {st }}$ degree kin with dementia); ${ }^{9,10,19,20}$ prescription and over-the-counter medications, particularly anticholinergics; ${ }^{16,20,21,24}$ scales of depression and caregiver burden; ${ }^{14,17,21}$ and measures of life style and health behaviors (included by 10 studies). No study included all these areas.

\section{Findings}

The impact of oral health on cognitive status (11 articles)-Two studies indicated that having fewer teeth at baseline or decline in number of teeth, was not associated significantly with cognitive decline. ${ }^{11,20}$ This was contradicted by other studies which found an association with cognitive decline, ${ }^{10,16}$ and with dementia. ${ }^{14,15,23}$ Findings are further complicated: one reported an association only in women; ${ }^{19}$ another reported that with higher education the number of missing teeth was not associated with dementia, but among those with lower education, individuals with a greater number of missing teeth were less likely to have dementia; ${ }^{17}$ and a third reported that having fewer teeth increased the hazard of dementia, but not when periodontal damage was present. ${ }^{9}$ Development of caries or the presence of new restoration was associated with lower performance on the MMSE and a spatial copying task. ${ }^{16}$ Mastication difficulty was not associated with incident dementia. ${ }^{23}$

Five studies examined the effect of periodontal disease on incident dementia, cognitive decline, or low level of cognitive functioning. ${ }^{9,14,16,17,20}$ Two of them found some indication of a relationship: alveolar bone loss and pocket depth was associated with poor cognitive functioning, ${ }^{16}$ the other found a significant decline on the Modified Mini-Mental State (3MS) Examination (which assesses several areas of cognition), but only when the continuous form of the gingival index was categorized, and the bottom quartile compared with the rest; no significant decline was found on the Digit Symbol Substitution Test. ${ }^{20}$

Three studies asked about or provided personal dental care. An intervention in nursing homes to make sure that teeth, dentures, and the oral cavity were cleaned, and plaque and calculus controlled, found a significantly smaller decline in MMSE score in the group receiving this oral care, however, variation was large and the numbers present at study end were unclear. ${ }^{12}$ In one study, poor oral hygiene and no regular visits to the dentist predicted 
incident dementia. ${ }^{23}$ Similar findings were reported in another study but were sex-specific -no daily tooth brushing (women only), and no dental visits in the previous 12 months (men only) predicted incident dementia. ${ }^{19}$

The impact of cognitive status on oral health ( 5 articles)-Sample sizes tended to be small when examining the impact of cognitive status on longitudinal oral status, while the number of oral outcomes of interest was large. One study, which provided routine dental care and treatment, focused specifically on tooth loss; neither number of teeth lost, nor rate of loss differed significantly between the dementia and no dementia groups. ${ }^{24}$ Other studies also found no statistically significant differences in number of teeth or restored surfaces. ${ }^{13,22}$

Considerable attention was paid to increase in the number of caries, which, variously, was higher in patients with dementia, ${ }^{21}$ higher but not significant because of small sample size, ${ }^{8}$ or not significantly different. ${ }^{22}$ Poorer MMSE scores were associated with increased gingival bleeding and plaque and diminished mucosal health, but with small sample size these findings were only suggestive. ${ }^{12,14,16}$

\section{Assessment of strength of the eligible studies}

We used a set of criteria to assess each of the studies, including sample selection, adequate sample size, description of the sample, validated assessment of the exposure (e.g., cognitive status or oral health measure) and outcome (e.g., cognitive status or oral health measure), length of follow-up period, attrition, controls for confounding, and appropriate analytic approach. ${ }^{40}$ The evidence suggests that there are methodological deficiencies in this area, most notably due to small sample size or lack of representativeness of the population, inadequate assessment of cognitive function and lack of clinical evaluation of oral health. Thus, there was insufficient evidence to date for a causal association between cognitive function and oral health.

\section{DISCUSSION}

Our systematic review of the first two decades of longitudinal studies designed to examine the association between oral health and cognitive status identified 16 studies, 11 examined the effect of oral status on change in cognitive status, and five the reverse. The uneven distribution likely reflects the costs and difficulties of carrying out oral health assessments. It is easier and cheaper to add cognitive assessments to a study for which oral health has already been measured, than to do the reverse.

It is currently unclear how or whether oral health conditions and cognitive status are related. Findings based on number or change in number of teeth or caries, are conflicting, or, because of inadequate sample size, do not reach statistical significance. Limited studies (two out of five) found periodontal conditions, such as gingival health or pocket depth, was associated with poorer cognitive status or cognitive decline, with findings possibly sensitive to the neuropsychology assessments used. There are indications, albeit weak, that better oral hygiene and regular dental visits may reduce the rate of cognitive decline, and the hazard of incident dementia. ${ }^{12,19,23}$ Certainly, with good dental care, the dental status of patients with dementia remained comparable to that of patients without a dementing disorder. ${ }^{24}$ These 
findings may reflect that incipient cognitive decline or dementia leads to decline in hygiene, but that oral health can be maintained with self or assisted oral hygiene, and dental appointments.

While the argument of a common underlying cause associated with inflammation may be enticing, ${ }^{1,2}$ it was examined directly in only one study, and there found to be only marginally relevant. ${ }^{20}$ Current studies are not adequate to indicate whether poor oral health and cognitive decline have a common underlying cause in inflammation, but with the increase in major surveys with biomarker data, test of this hypothesis is becoming more feasible.

Because of the costs of detailed dental examination, there may be a temptation to carry out small sample studies. As seen in the current review, this results in a lack of power. There is also a temptation to match cases and controls, but matching must be considered carefully. Doing so eliminates the possibility of examining the "matching" conditions (e.g., demographic characteristics), which may be associated with both oral health and cognitive status. With an older population, the duration of a study becomes an issue because of incidence of additional chronic conditions which may affect oral health and cognition, attrition due to sickness, and death.

To better compare findings across studies, agreement is needed regarding the oral health issues to be explored, oral health and neuropsychological measures to use, and covariates for which to adjust. In particular, to reduce the costs of dental studies, additional information is needed on the extent to which self-report accurately determines oral health condition, oral hygiene behavior, and dental visits. While it appears that self-assessment of obvious conditions (e.g., number of teeth), and those that are salient (e.g., root canal, dentures) can be reported reliably (with accuracy varying by demographic status), report is less reliable for conditions requiring oral health expertise (e.g., caries, periodontal disease). ${ }^{32,33}$ However, since no studies have been conducted to examine the reliability of self-reported data among individuals with cognitive impairment, we are less certain how reliable the self-reported oral health data is in such individuals.

There is a considerable literature on assessment of cognitive state, with agreement on preferred measures to use in Memory Disorders clinics to assess dementing disorders, ${ }^{34}$ and procedures to follow in epidemiological studies, such as diagnostic adjudication. With increasing information on dementia, diagnostic criteria are changing, and diagnosis at an earlier stage of disease is becoming possible. Consequently, the characteristics of diagnosed subjects may change, with findings on an oral health/dementia association changing also.

Standardization of assessment of cognitive status should be considered. The most frequently used measure has been the MMSE, ${ }^{28}$ indicating preference for a brief, easy to administer assessment. The MMSE has several drawbacks: multiple versions with unknown equivalence; performance, as with many brief cognitive screens, that is age-, race-, and education-biased; a ceiling effect, and payment required for use. Improved screens, such as the Montreal Cognitive Assessment ${ }^{35}$ have become available since data were gathered for many of the current studies, and these screens should be considered. Agreement on use of a brief screen would facilitate comparison of findings, and aggregation of data. 
Covariates varied from study to study, with a large variety reported: demographic, health conditions (in particular cardiovascular conditions and risk factors), medications (anticholinergics), health behaviors, and an assortment of other characteristics (e.g., scales of depression, caregiver burden). Certain relevant information was rarely included: e.g., nutrition, which affects oral health and general health, and environmental characteristics (dental insurance, access to dentists, cultural expectations regarding dentition). Although many studies have found self-report of medical conditions to be valid, ${ }^{36}$ caution is needed regarding medical and dental records and administrative data. Diagnosis of dementia based on administrative records which determine level of care to be provided may yield overdiagnosis, while dementing disorders may be under-reported in administrative medical records and death certificates. ${ }^{37,38}$ Agreement is needed on which covariates to include in analysis, and the manner in which they should be handled, in particular, regarding categorization of age, and education.

Current studies that capitalize on already available information on oral health, cognition, or biomarkers, illustrate how sample size and possibly representativeness can be increased, and costs reduced. In addition to secondary analysis of data, ${ }^{14,16,20}$ some studies have used administrative records with known sensitivity and specificity for diagnosis of dementia, ${ }^{15}$ while others have linked the main study data to dental records. ${ }^{9,10}$ Although there is no report as to whether those dental records were evaluated, studies that have examined preexisting radiographs have found them to be usable for assessment of periodontal disease. ${ }^{39}$

This is the first systematic review to examine longitudinal studies focused on oral health and cognition. As such, it shows substantial diversity across studies with regard to data and sample sources, sample size, and variety of oral health data and cognitive data obtained. In summary, the strength of the evidence is weak, and findings were often inconsistent. For example, some studies found a significant association, albeit relatively weak, between periodontal disease and cognitive decline; but other studies found no association. Similarly the association between dental caries and cognition was inconsistent across studies. Findings are even more complex for the association between tooth loss and cognitive decline, ranging from a positive association, to no association, to a negative association (only in the group with a lower level of education) across different studies. However, these studies lay the groundwork for future longitudinal work.

While research in the field appears to be moving in the right direction, we would like to make some specific suggestions, operationalization of which may permit more accurate determination of the grounds for an association, if one is present, between oral health and cognitive status. In the current studies, cognitive function has been evaluated using a great variety of cognitive measures. Information could be better evaluated if there was agreement on a uniform set of cognitive assessments. When change in cognition is assessed, it may be preferable to examine decline in cognitive function (which is more straightforward to assess), instead of development of dementing disorders, with attendant complexities in diagnosis. Regarding the assessment of oral health status, several studies used standardized oral health examination protocols, such as the U.S. National Institute of Dental Research protocol, ${ }^{21,22}$, a procedure which should be emphasized. Future research should pay more attention to calibration of dental assessment, both between and within dental evaluators. The 
oral health outcomes used should reflect oral health status which can change during the study period. With respect to other aspects of study design, use of standard covariates, samples sized adequately for the number and type of outcomes to be measured and better representative of the study population, a study duration appropriate to the planned outcome, and sophisticated statistical approaches already in use should be encouraged.

\section{Supplementary Material}

Refer to Web version on PubMed Central for supplementary material.

\section{Acknowledgments}

Funding sources: This study was supported by NIH/NIDCR grant number 1R01DE019110 (BW), NIH/NINR grant number 1P30NR014139 (BW), and NIH/NIA grant number 1P30AG028716 (GGF). This study was presented at the annual scientific meeting of the Gerontological Society of America, 2013. We would like to thank Qin Lu for his assistance with the literature review.

Sponsor's role: No sponsor was involved in the design, methods, subject recruitment, data collections, analysis or preparation of this paper.

\section{REFERENCES}

1. Kamer AR, Craig RG, Dasanayake AP, et al. Inflammation and Alzheimer's disease: Possible role of periodontal diseases. Alzheimers Dement. 2008; 4:242-250. [PubMed: 18631974]

2. Noble JM, Scarmeas N, Papapanou PN. Poor oral health as a chronic, potentially modifiable dementia risk factor: Review of the literature. Curr Neurol Neurosci Rep. 2013; 13:1-8.

3. Wu B, Liang J, Landerman L, et al. Trends of edentulism among middle-aged and older Asian Americans. Am J Public Health. 2013; 103:e76-e82. [PubMed: 23865668]

4. Plassman BL, Langa KM, Fisher GG, et al. Prevalence of cognitive impairment without dementia in the United States. Ann Intern Med. 2008; 148:427-434. [PubMed: 18347351]

5. Plassman BL, Langa KM, Fisher GG, et al. Prevalence of dementia in the United States: The aging, demographics, and memory study. Neuroepidemiology. 2007; 29:125-132. [PubMed: 17975326]

6. Daviglus M, Bell C, Berrettini W, et al. NIH State-of-the-Science Conference Statement: Preventing Alzheimer's disease and cognitive decline. NIH Consensus and State-of-the-Science Statements. 2010; 27:1-30. [PubMed: 20445638]

7. Hebert LE, Weuve J, Scherr PA, et al. Alzheimer disease in the United States (2010-2050) estimated using the 2010 census. Neurology. 2013; 80:1778-1783. [PubMed: 23390181]

8. Jones JA, Lavallee N, Alman J, et al. Caries incidence in patients with dementia. Gerodontology. 1993; 10:76-82. [PubMed: 7713530]

9. Stein PS, Desrosiers M, Donegan SJ, et al. Tooth loss, dementia and neuropathology in the Nun study. J Am Dent Assoc. 2007; 138:1314-1322. [PubMed: 17908844]

10. Stein P, Kryscio R, Desrosiers M, et al. Tooth loss, apolipoprotein E, and decline in delayed word recall. J Dent Res. 2010; 89:473-477. [PubMed: 20139337]

11. Shimazaki Y, Soh I, Saito T, et al. Influence of dentition status on physical disability, mental impairment, and mortality in institutionalized elderly people. J Dent Res. 2001; 80:340-345. [PubMed: 11269726]

12. Yoneyama T, Yoshida M, Ohrui T, et al. Oral care reduces pneumonia in older patients in nursing homes. J Am Geriatr Soc. 2002; 50:430-433. [PubMed: 11943036]

13. Ship JA, Puckett SA. Longitudinal study on oral health in subjects with Alzheimer's disease. J Am Geriatr Soc. 1994; 42:57-63. [PubMed: 8277117]

14. Batty GD, Li Q, Huxley R, et al. Oral disease in relation to future risk of dementia and cognitive decline: Prospective cohort study based on the Action in Diabetes and Vascular Disease: Preterax 
and Diamicron Modified-Release Controlled Evaluation (ADVANCE) trial. Eur Psychiatry. 2013; 28:49-52. [PubMed: 21964484]

15. Gatz M, Mortimer JA, Fratiglioni L, et al. Potentially modifiable risk factors for dementia in identical twins. Alzheimers Dement. 2006; 2:110-117. [PubMed: 19595867]

16. Kaye EK, Valencia A, Baba N, et al. Tooth loss and periodontal disease predict poor cognitive function in older men. J Am Geriatr Soc. 2010; 58:713-718. [PubMed: 20398152]

17. Arrivé E, Letenneur L, Matharan F, et al. Oral health condition of French elderly and risk of dementia: A longitudinal cohort study. Community Dent Oral Epidemiol. 2012; 40:230-238. [PubMed: 22059867]

18. Batty GD, Li Q, Czernichow S, et al. Erectile dysfunction and later cardiovascular disease in men with type 2 diabetes prospective cohort study based on the ADVANCE (Action in Diabetes and Vascular Disease: Preterax and Diamicron Modified-Release Controlled Evaluation) Trial. J Am Coll Cardiol. 2010; 56:1908-1913. [PubMed: 21109113]

19. Paganini-Hill A, White SC, Atchison KA. Dentition, dental health habits, and dementia: The Leisure World Cohort Study. J Am Geriatr Soc. 2012; 60:1556-1563. [PubMed: 22860988]

20. Stewart R, Weyant RJ, Garcia ME, et al. Adverse oral health and cognitive decline: The health, aging and body composition study. J Am Geriatr Soc. 2013; 61:177-184. [PubMed: 23405916]

21. Chalmers JM, Carter KD, Spencer AJ. Caries incidence and increments in community-living older adults with and without dementia. Gerodontology. 2002; 19:80-94. [PubMed: 12542217]

22. Ellefsen B, Holm-Pedersen P, Morse DE, et al. Assessing caries increments in elderly patients with and without dementia: A one-year follow-up study. J Am Dent Assoc. 2009; 140:1392-1400. [PubMed: 19884398]

23. Yamamoto T, Kondo K, Hirai H, et al. Association between self-reported dental health status and onset of dementia: A 4-year prospective cohort study of older Japanese adults from the Aichi Gerontological Evaluation Study (AGES) Project. Psychosom Med. 2012; 74:241-248. [PubMed: 22408130]

24. Chen X, Shuman SK, Hodges JS, et al. Patterns of tooth loss in older adults with and without dementia: A retrospective study based on a Minnesota cohort. J Am Geriatr Soc. 2010; 58:2300 2307. [PubMed: 21143439]

25. Snowdon DA, Greiner LH, Mortimer JA, et al. Brain infarction and the clinical expression of Alzheimer disease: The Nun Study. JAMA. 1997; 277:813-817. [PubMed: 9052711]

26. Reisberg B, Ferris SH, de Leon MJ, et al. The Global Deterioration Scale for assessment of primary degenerative dementia. Am J Psychiatry. 1982; 139:1136-1139. [PubMed: 7114305]

27. Braak H, Braak E. Neuropathological stageing of Alzheimer-related changes. Acta Neuropathol. 1991; 82:239-259. [PubMed: 1759558]

28. Folstein MF, Folstein SE, McHugh PR. "Mini-mental state": A practical method for grading the cognitive state of patients for the clinician. J Psychiatr Res. 1975; 12:189-198. [PubMed: 1202204]

29. Dartigues JF, Gagnon M, Barberger-Gateau P, et al. The Paquid epidemiological program on brain ageing. Neuroepidemiology. 1992; 11(Suppl. 1):14-18. [PubMed: 1603241]

30. Morris J, Heyman A, Mohs R, et al. The Consortium to Establish a Registry for Alzheimer's. Disease (CERAD). Part I. Clinical and neuropsychological assesment of Alzheimer's disease. Neurology. 1989; 39:1159-1165. [PubMed: 2771064]

31. Charlson ME, Pompei P, Ales KL, et al. A new method of classifying prognostic comorbidity in longitudinal studies: Development and validation. J Chronic Dis. 1987; 40:373-383. [PubMed: 3558716]

32. Liu H, Maida CA, Spolsky VW, et al. Calibration of self-reported oral health to clinically determined standards. Community Dent Oral Epidemiol. 2010; 38:527-539. [PubMed: 21054482]

33. Pitiphat W, Garcia RI, Douglass CW, et al. Validation of self-reported oral health measures. J Public Health Dent. 2002; 62:122-128. [PubMed: 11989207]

34. Weintraub S, Salmon D, Mercaldo N, et al. The Alzheimer's Disease Centers' uniform data set (UDS): The neuropsychological test battery. Alzheimer Dis Assoc Disord. 2009; 23:91-101. [PubMed: 19474567] 
35. Nasreddine ZS, Phillips NA, Bédirian V, et al. The Montreal Cognitive Assessment, MoCA: A brief screening tool for mild cognitive impairment. J Am Geriatr Soc. 2005; 53:695-699. [PubMed: 15817019]

36. Baumeister H, Kriston L, Bengel J, et al. High agreement of self-report and physician-diagnosed somatic conditions yields limited bias in examining mental-physical comorbidity. J Clin Epidemiol. 2010; 63:558-565. [PubMed: 19959329]

37. Taylor DH Jr, Fillenbaum GG, Ezell ME. The accuracy of medicare claims data in identifying Alzheimer's disease. J Clin Epidemiol. 2002; 55:929-937. [PubMed: 12393082]

38. Romero JP, Benito-León J, Louis ED, et al. Under reporting of dementia deaths on death certificates: A systematic review of population-based cohort studies. J Alzheimers Dis. 2014; 41:213-221. [PubMed: 24583403]

39. Pitiphat W, Crohin C, Williams $\mathrm{P}$, et al. Use of preexisting radiographs for assessing periodontal disease in epidemiologic studies. J Public Health Dent. 2004; 64:223-230. [PubMed: 15562945]

40. Williams, JW.; Plassman, BL.; Burke, J., et al. Preventing Alzheimer's disease and cognitive decline. Evidence Report/Technology Assessment No. 193 (Prepared by the Duke Evidence-based Practice Center under Contract No. HHSA 290-2007-10066-I.) AHRQ Publication No. 10-E005. Rockville, MD: Agency for Healthcare Research and Quality; 2010 Apr. 


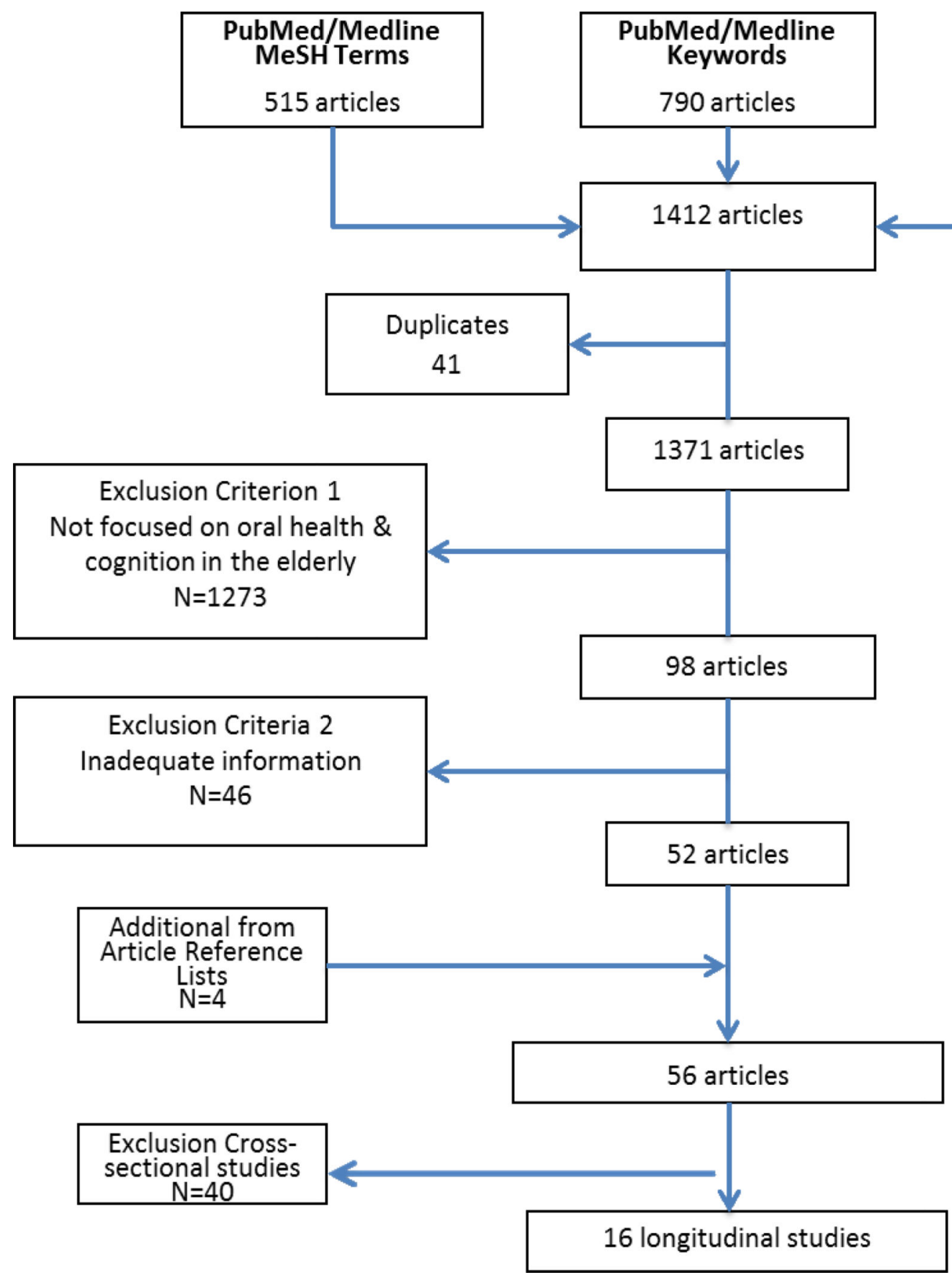

Figure 1.

Identification of relevant articles 


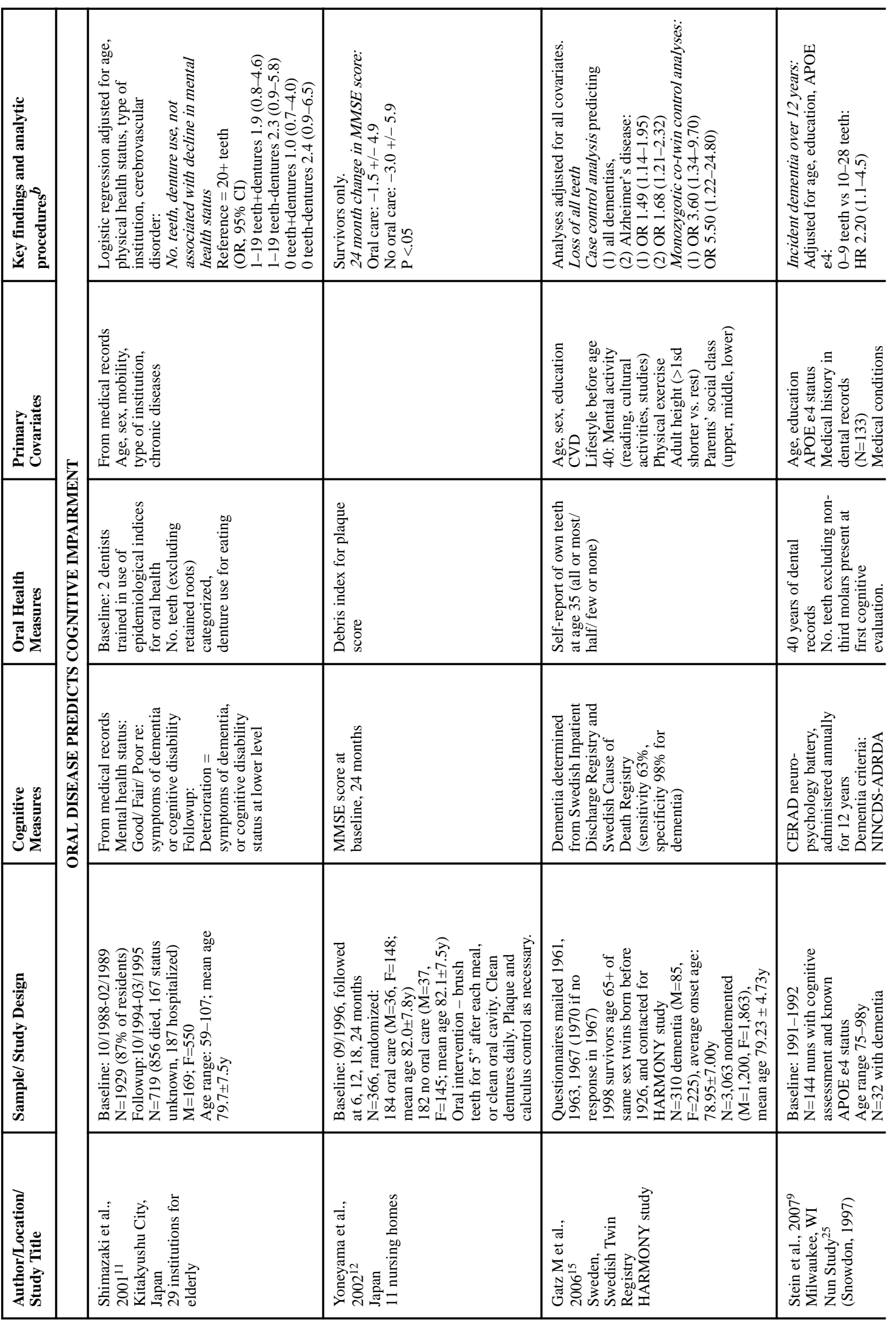

J Am Geriatr Soc. Author manuscript; available in PMC 2017 April 01. 


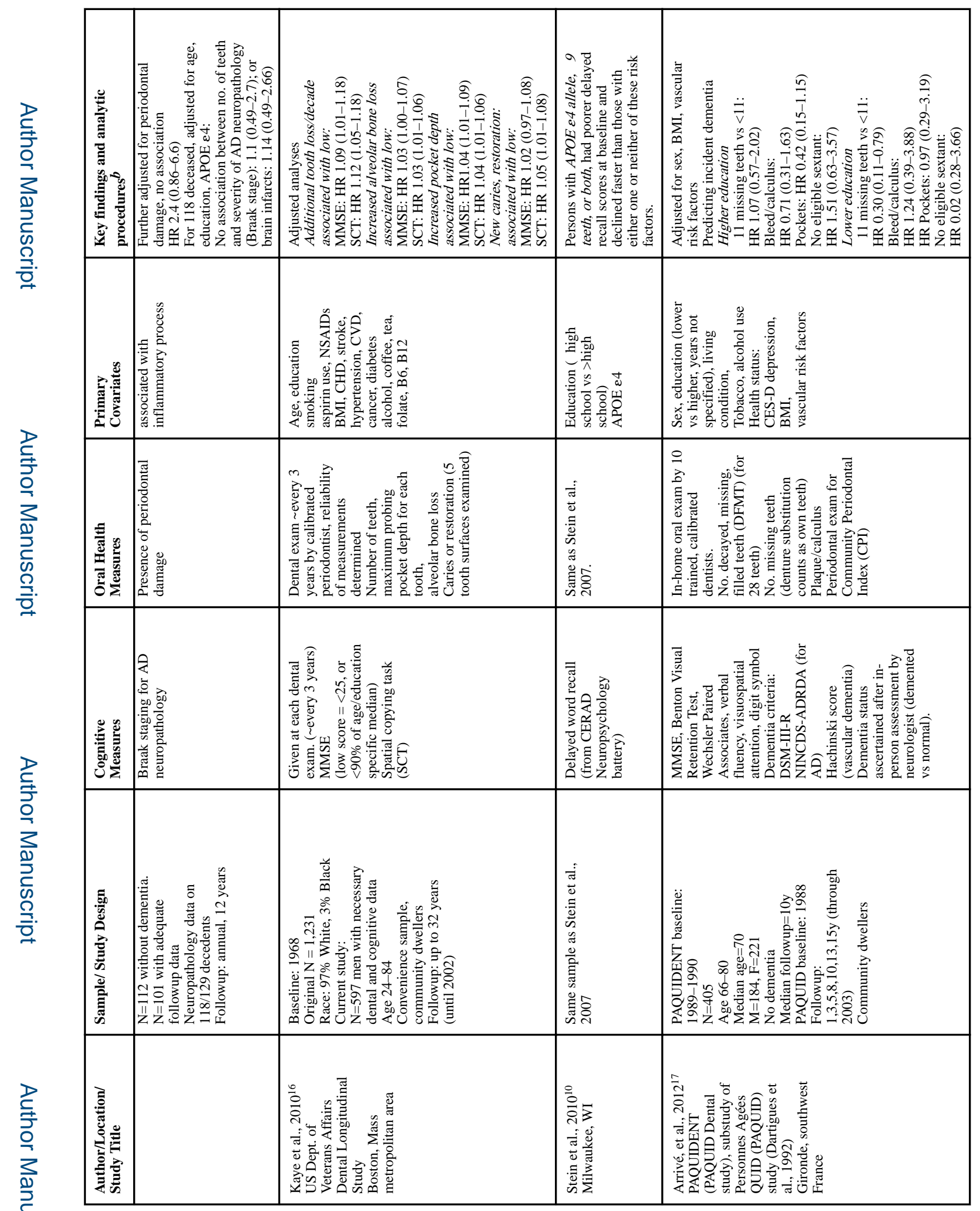

J Am Geriatr Soc. Author manuscript; available in PMC 2017 April 01. 


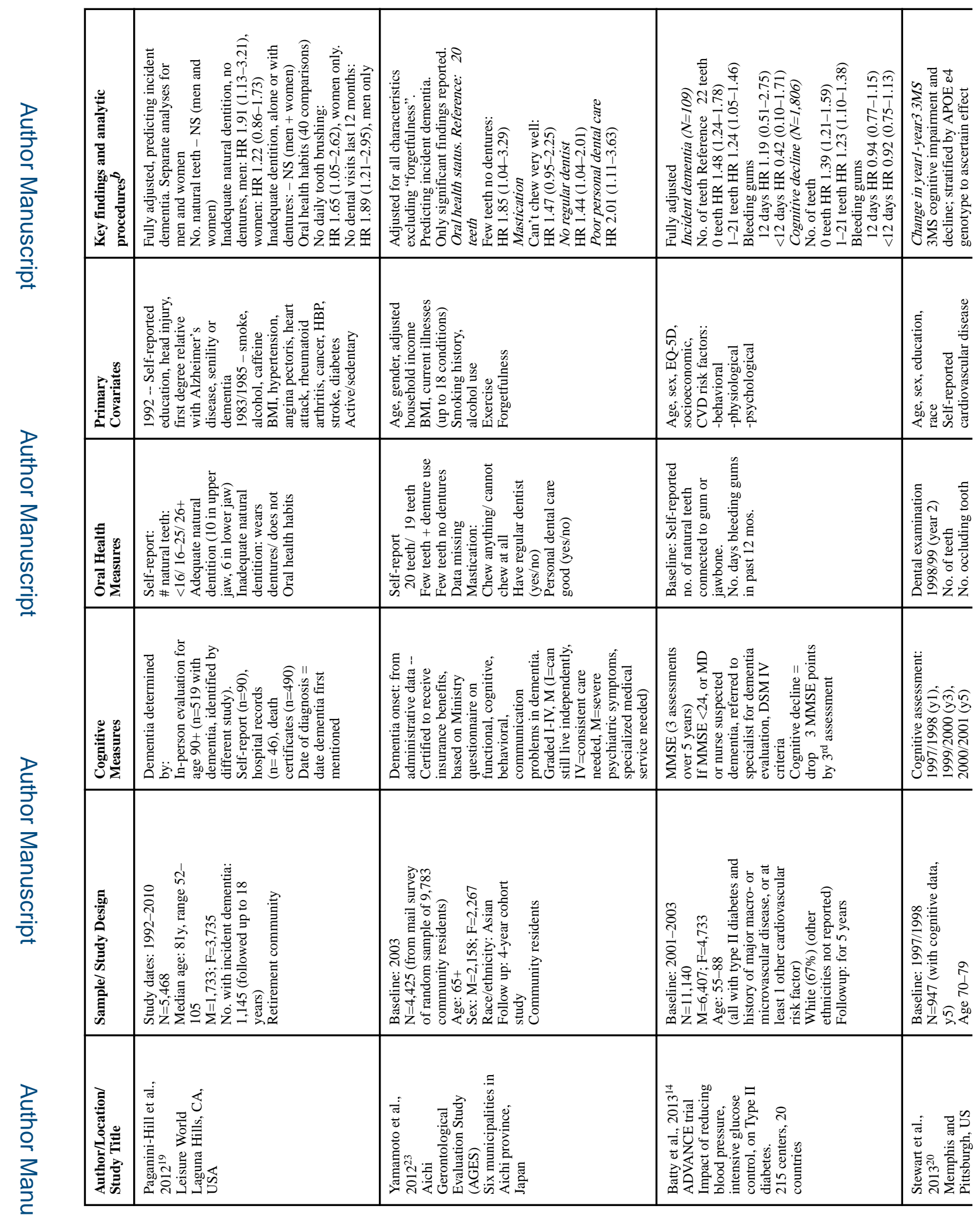

J Am Geriatr Soc. Author manuscript; available in PMC 2017 April 01. 


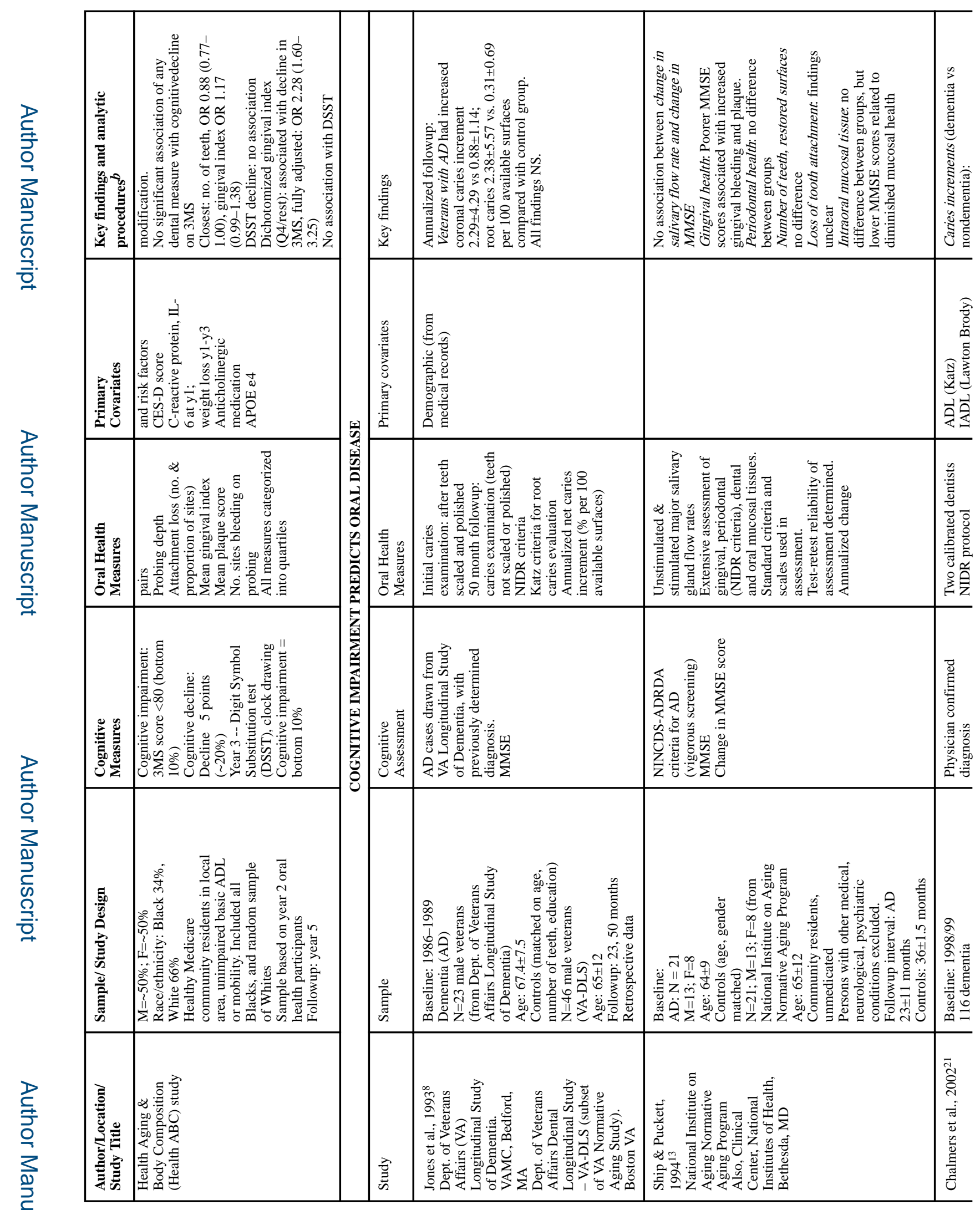

J Am Geriatr Soc. Author manuscript; available in PMC 2017 April 01. 


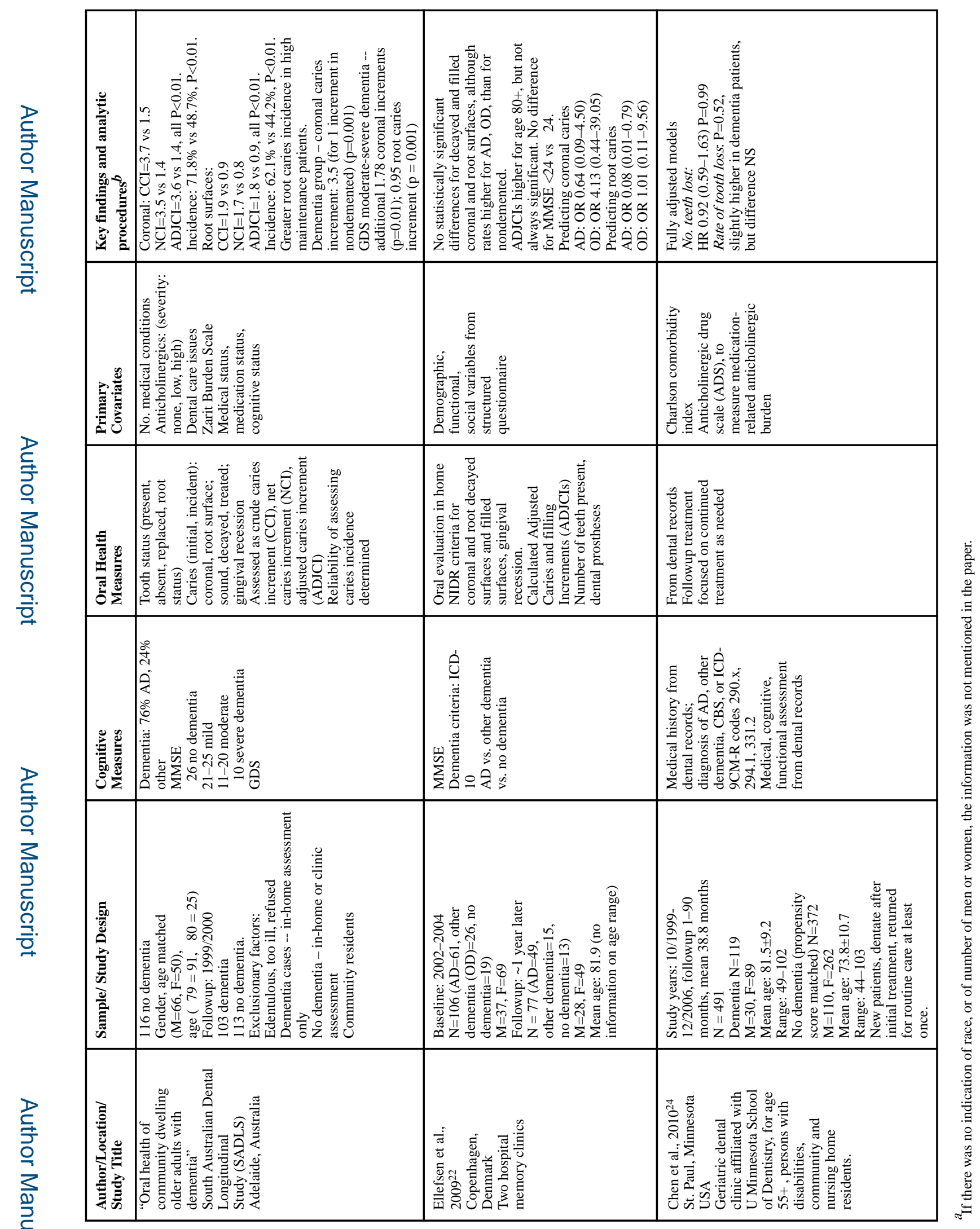

J Am Geriatr Soc. Author manuscript; available in PMC 2017 April 01. 


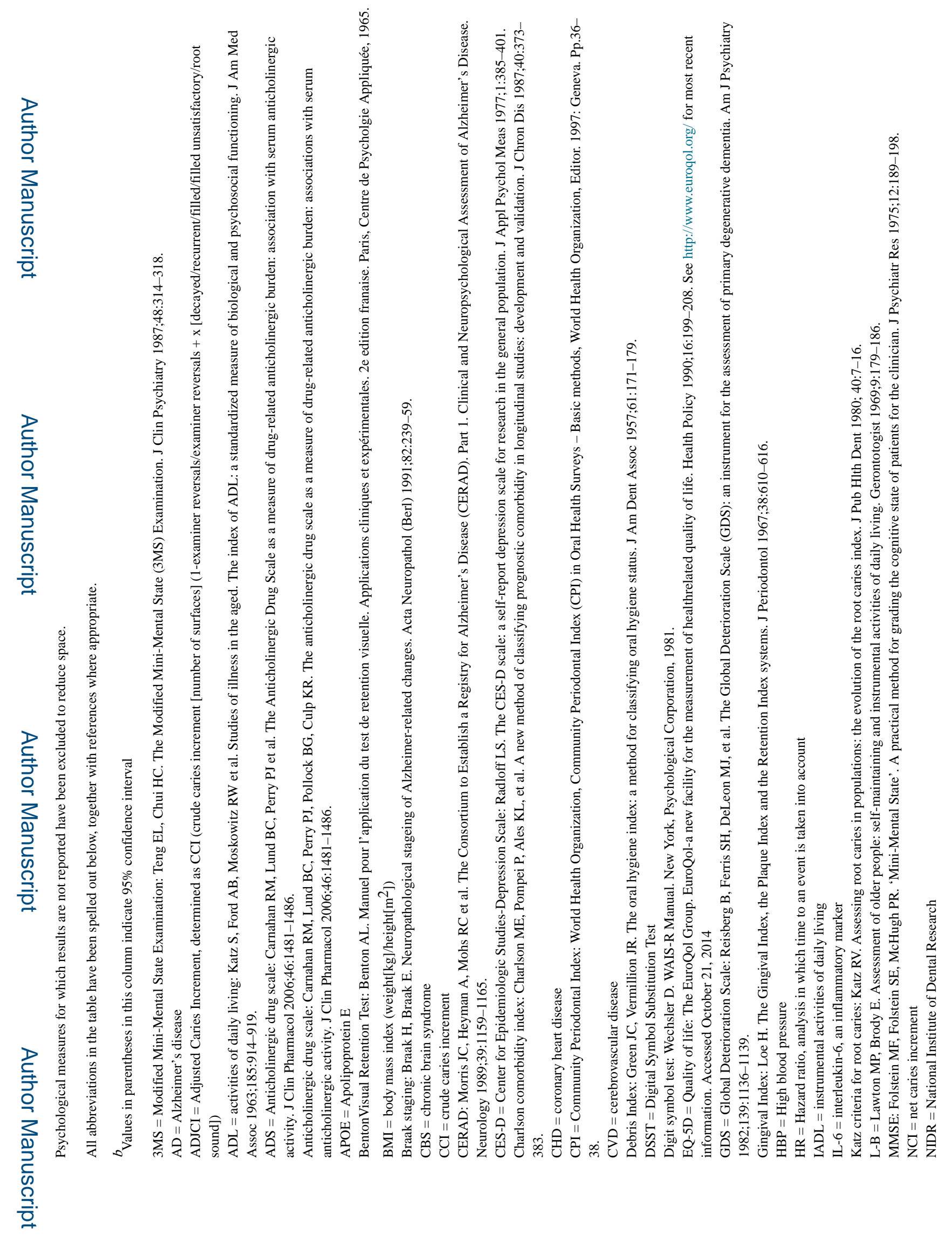

J Am Geriatr Soc. Author manuscript; available in PMC 2017 April 01. 


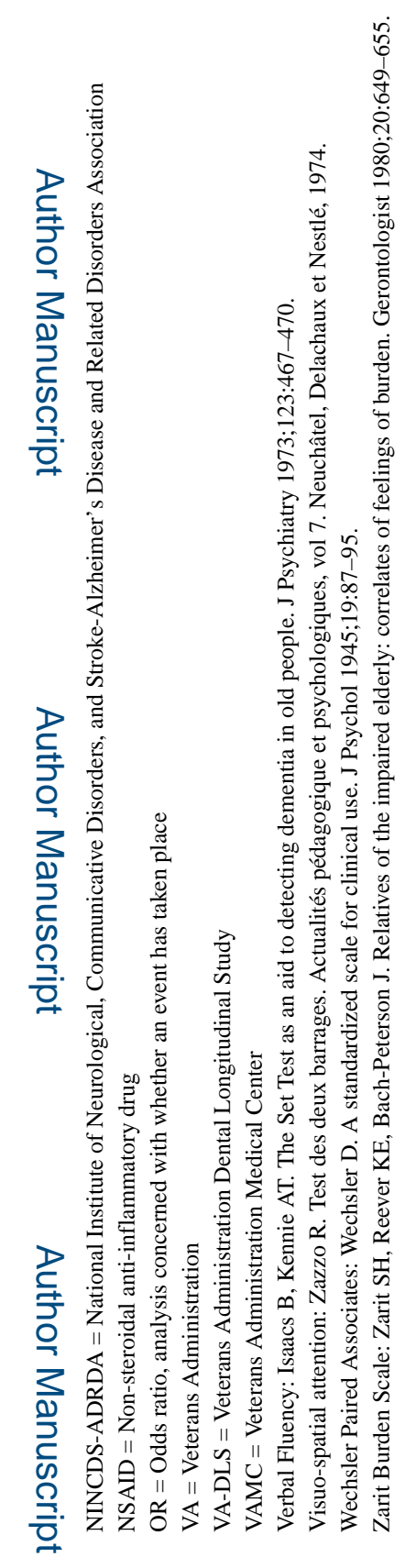

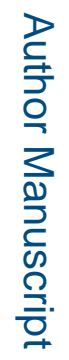

